\title{
Digitlab: tecnologías emergentes y ambientes de aprendizaje mediado por tecnologías para fortalecer habilidades de pensamiento y comunicación en las disciplinas del diseño
}

institucional.us.es/ambitos/

\section{Silvia Husted Ramos}

Universidad Autónoma de Ciudad Juárez

shusted@uacj.mx

\author{
Gloria Olivia Rodríguez Garay \\ Universidad Autónoma de Ciudad Juárez \\ grodrigu@uacj.mx
}

\section{Martha Patricia Álvarez Chávez \\ Universidad Autónoma de Ciudad Juárez malvarez@uacj.mx}

English Version: ...

\section{Resumen}

La tecnología digital emergente dinamiza la educación universitaria, con este objetivo se creó elDigitLAB un laboratorio inspirado en el Movimiento "Maker", para fortalecer conocimiento, prácticas profesionales y aprendizaje acerca de tecnologías digitales para el diseño con tecnología telemática. DigitLAB atiende diversos contextos educativos aplicando herramientas informáticas, procesos dialógicos y

comunicaciones interactivas. Este trabajo muestra tres diferentes escenarios de aprendizaje generados de este proyecto y resultados de las prácticas comunicativas que se desarrollan a través de herramientas telemáticas. Se evalúan las tecnologías

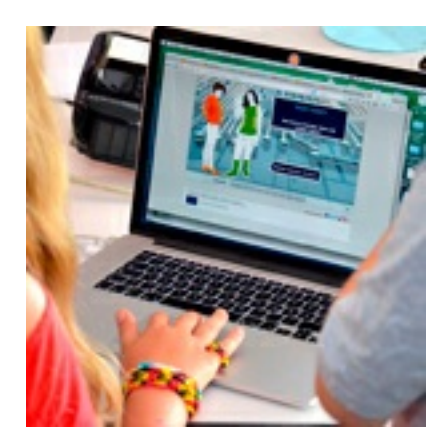
emergentes en la práctica del diseño interdisciplinar y la comunicación efectiva en

ambientes virtuales de trabajo colaborativo.

\section{Palabras clave}

Makerspace, Diseño interdisciplinar, Herramientas telemáticas, Tecnologías emergentes, Comunicación interactiva.

The emerging digital technology streamlines university education, this goal was established as the laboratory DigitLAB to strengthen knowledge, professional practice and learning about digital technologies to design with telematics technology. DigitLAB heeds several educational contexts using computing's tools, dialogic processes and interactive communications. This work shows three different learning scenarios generated from this project and results of communicative practices that develop through telematics tools. Assesses emerging technologies in the practice of interdisciplinary design and effective communication in virtual collaborative work environments.

Keywords

Makerspace, Interdisciplinarydesign, Telematics tools, Emerging Technologies, Interactive Communication. 


\section{INTRODUCCIÓN}

Un problema que aqueja a las disciplinas del diseño en la actualidad tiene que ver con la interdisciplinariedad, Fragoso (2008) asegura que las disciplinas del diseño se proclaman independientes, por alguna razón desde una perspectiva de la comunicación los fenómenos del diseño se abordan con el enfoque de una sola disciplina aun cuando en el campo del diseño teorías y métodos se conceptúan desde la óptica de una actividad multidisciplinaria.

El diseño es una disciplina muy dinámica y en constante transformación, esto se debe al desarrollo de la tecnología, los cambios sociales y del entorno actual. Sin embargo, e incluso con las transformaciones tecnológicas actuales y la diversificación, la disciplina sigue sin entender la realidad del diseño como un conjunto de problemas que interactúan entre sí en un contexto cambiante (Juez 2002). Por lo tanto, la interdisciplinariedad en el diseño debe formalizarse desde el comienzo de la formación universitaria como referencia para la futura práctica profesional (Tresserras, 2015).

Con base en lo anterior, podemos decir que hoy en día las escuelas de diseño exigendistintos escenarios para la educación y estrategias que apoyen las nuevas formas de adquirir conocimiento, habilidades y competencias. Con la idea de esto se crea el proyecto "Makerspace: espacio interdisciplinar para el Diseño", sustentándose en la potencialidad de tecnologías para la comunicación y la productividad como los componentes clave para la introducción a un ambiente de aprendizaje basado en la interdisciplinariedad y bajo la filosofía del movimiento "Maker".

\section{EL MOVIMIENTO “MAKER” COMO MOTIVACIÓN CREATIVA DEL DIGITLAB}

El movimiento "Maker" es iniciado en Viena por Paul Bohm en el 2006, no obstante de acuerdo a Blikstein y Krannich (2013) la idea detrás del movimiento no es nueva, se sitúa tres décadas atrás y sus pilares teóricos y pedagógicos provienen del constructivismo, la educación experimental y la pedagogía crítica. A una década de su resurgimiento se considera el más reciente impulso de democratización de la tecnología y se establece como un recurso muy potente para construir una nueva dinámica de interacción entre el ser humano y la tecnología (Tesconi y Arias, 2015).

Actualmente los Makerspace se han expandido y diversificado, un laboratorio de aprendizaje de este tipopuede ser denominado"hakerspace", "makerspace" o "makerEd",según su orientación, sin embargotodosrefieren a un espacio que provee las herramientas necesarias para "hacer", se diseñan parapersonas de todas las edadesy pueden encontrarse en contextos informales (locales o casas particulares), formales (bibliotecas e instituciones educativas) o apoyarse de plataformas digitales (sitios web). Su característica principal es la tecnología y el uso de diferentes medios de comunicación quepermitenla amplitud, profundidad y conexión de experiencias e intereses (Ito, Gutiérrez, Livingstone, Penuel, Rhodes, Salen y Watkins, 2013).

En todas estasderivaciones prevalece la filosofía "Maker"que consiste endemocratizar el acto de hacer algo desde cero, con la idea de que cualquier persona debe ser capaz de hacer cualquier cosa en cualquier momento con cualquier material disponible, solo o con la ayuda de otros (Ito, et al., 2013).

En cuanto a la escuela, los MakerEdhan venido a refrescar la idea que se tenía del laboratorio ya que pueden responderal entorno y la situación donde se generancomo ambientes de aprendizaje,además de ajustarse a diversos propósitos educativos. Blikstein y Krannich(2013) mencionan que es necesario pensar en nuevas formas de diseñar contextos de aprendizaje desde esta perspectiva como un medio para comprender cómo los actores experimentan estos espacios de creación.

\section{- Elementos y tendencias de un Makerspace educativo (MakerEd)}

En el contexto educativo se presentan características específicas del Makerspace que pueden apreciarse a través de las naturalezas que lo integran que según Tesconi y Arias(2015) y Vossoughi y Bevan (2014) los elementos que componen un Makerspace educativo son: 
- Infraestructura: espacio físico, virtual o ambos.

- Herramientas: tecnológicas, para la creación, la colaboración, comunicación y productividad.

- Ambiente de aprendizaje: abierto, que propicie la práctica, la interacción, el intercambio de ideas e información.

- Un proyecto.

Lo que conlleva a cubrir esos aspectos para poder efectuar las actividades en un Makerspace.

- Tendencias actuales de losMakerEd

De igual manera Tesconi y Arias(2015) y Vossoughi y Bevan (2014) señalan que las tendencias actuales de los MakerEd se establecen de la siguiente forma:

- Como herramienta formativa para la creación de empresa.

- Como acceso a la práctica profesional en ciencia, ingeniería, tecnología y diseño.

- Como práctica educativa basada en la indagación.

- Como puente interdisciplinario.

Esto permite afirmar de manera contundente que el Makerspace es un espacio para la formación profesional y entre disciplinas que facilitan el intercambio de conocimientos y el desarrollo proyectual; lo que consintió al diseño del DigitLAB como un laboratorio inspirado en ese movimiento con el propósito de robustecer el conocimiento, lasprácticas profesionales y el aprendizaje con respecto a tecnologías digitales y telemáticas para el diseño.

\subsection{El Modelo de aprendizaje del DigitLAB}

El diseño base del DigitLABdesarrollado en la Universidad Autónoma de Ciudad Juárez (México) se presenta como unMakerspaceeducativo (makerEd)en un ambiente de aprendizajeactivo y abiertocon fundamento en la perspectiva constructivista de David Jonassen (2000).El diseño se realizó con el modelo Entornos de Aprendizaje Constructivista(EAC) yla estrategia didáctica Aprendizaje Basado en Proyectosdel mismo autor.El laboratorio se implementóen un espacio físico apoyado a su vez con una plataforma virtual. En ambos espacios pueden participar estudiantes de diversas disciplinas del diseño, ya sea de nivel pregrado o posgrado, y los proyectos pueden ser individuales, grupales o colectivos.

Lewis (2015), Ito et al. (2013) y Knobel\&Lankshear(2010) aseguran que las actividades que ocurren en espacios digitales de creación pueden fortalecer una serie de habilidades y competencias, las cuales son ingredientes clave para la productividad y esta puede hacerse evidente en el producto final.En los tres proyectos se propició el aprendizaje autodirigido y la comunicación interactiva a través del uso de herramientas telemáticas para la productividad y la comunicacióncon el propósito de generar puentes de colaboración interdisciplinar, además el uso y gestión de tecnologías emergentes para el diseño digital y web.

\subsubsection{Características del Modelo EAC - Entornos de Aprendizaje Constructivista}

Jonassen (2000) asegura que el conocimiento no puede ser transmitido, este debe ser elaborado individual y socialmente por los estudiantes con base en su experiencia e interpretación del mundo.El modelo de Entornos de Aprendizaje Constructivista (EAC)proponeel aprendizaje a partir de un problema, una pregunta o un proyecto como núcleo del entorno de aprendizaje para lo cual se debe proveer al estudiante de un contexto, la representación del problema y la posibilidad de manipular libremente el espacio, además de ofrecer al aprendiz sistemas de interpretación y de apoyo intelectual derivado de su alrededor. 


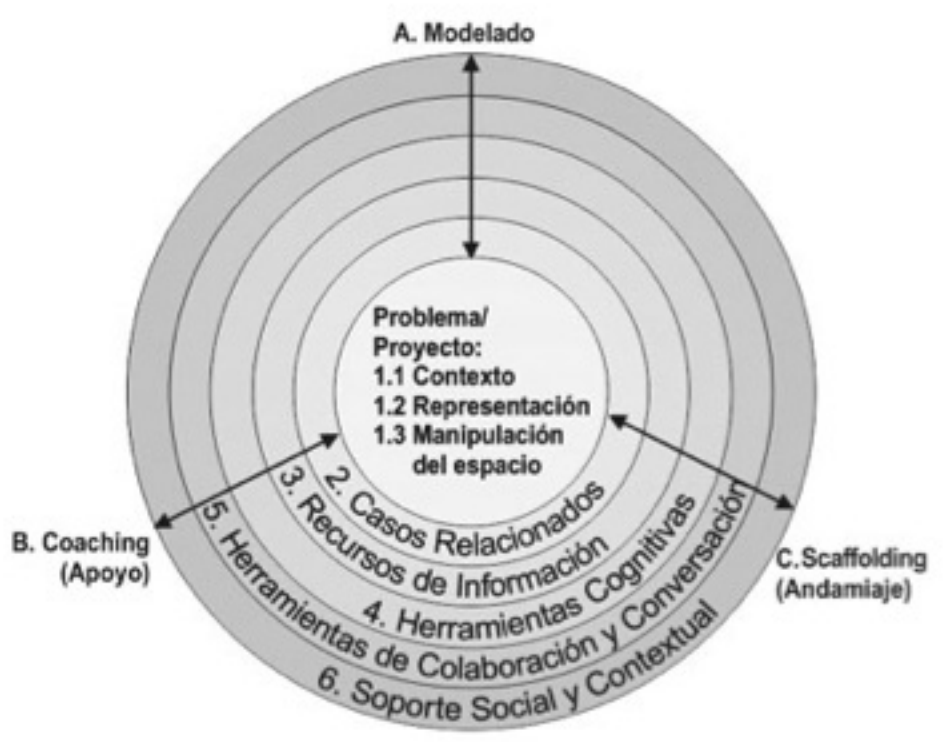

Figura No.1.El modelo EAC de DavidJonassen (2000). Elaboración propia.

\subsubsection{Elementos del Modelo EAC}

1. El proyecto/problema: Este puede ser generado por el profesor o por los mismos estudiantes y debe proporcionar:

1. Un contexto,

2. Espacio para la representación y

3. Posibilidad de manipular este espacio.

2. Casos relacionados o ejemplos: Se debe implementar un sistema para proporcionar ejemplos resueltos o casos relacionados para que el estudiante pueda guiarse de forma autónoma.

3. Recursos de información: Se debe propiciar o implementar un sistema para que el estudiante indague acerca de los conceptos que confieren al tema.

4. Herramientas cognitivas: Se deben facilitar herramientas para que el estudiante pueda documentar, organizar y exponer el material que se va generando con el proyecto.

5. Herramientas de Colaboración y Conversación: Se debe proporcionar un conjunto de herramientas de comunicación y conversación ya sea en su formato presencial o en virtual.

6. Soporte Social y contextual

1. Debe dar posibilidad al modelado (hacer, crear, inventar).

2. Debe proporcionarse apoyo (Coaching en caso de que el estudiante lo requiera).

3. Debe existir el andamiaje (personas con más experiencia que sirvan de guía y facilitador)

2.2.3.Los cuatro niveles de objetivos del ambiente de aprendizaje del DigitLAB 


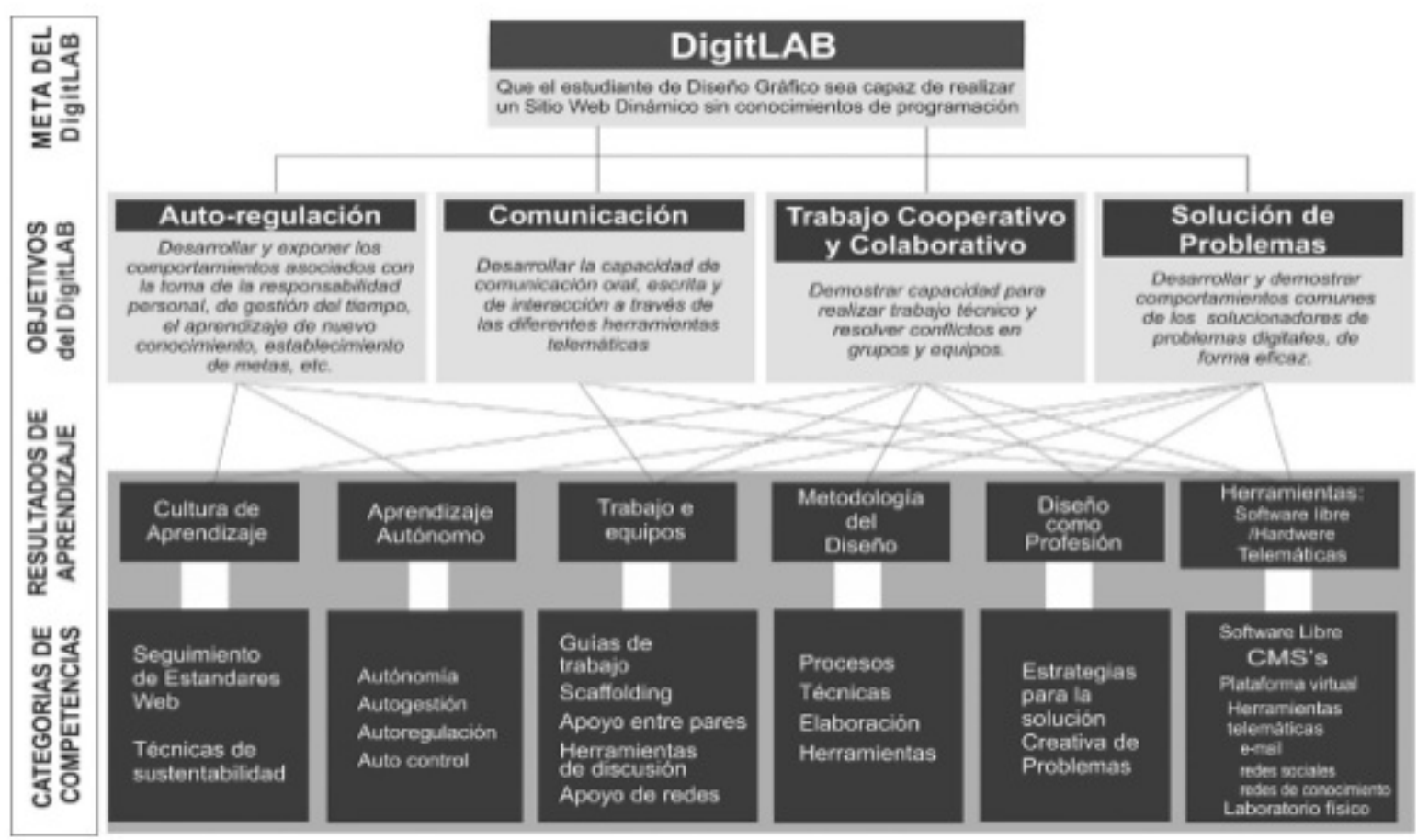

FiguraNo. 2.Los objetivos del DigitLAB. Elaboración propia.

\subsubsection{El Laboratorio DigitLAB}

El Digitlab está equipado con mesas de trabajo, equipo de cómputo, audio, video, fotografía,equipo para proyección,iluminación, tabletas para dibujo digital, una estación de trabajo para un facilitador (Scaffolding), una estación de trabajo para un Guía (Coaching), impresora, pantalla retráctil para proyección, pizarrón para lluvia de ideas y dibujo.

En las siguientes fotografías puede apreciarse el equipamiento tecnológico y actividades dentro del DigitLAB.
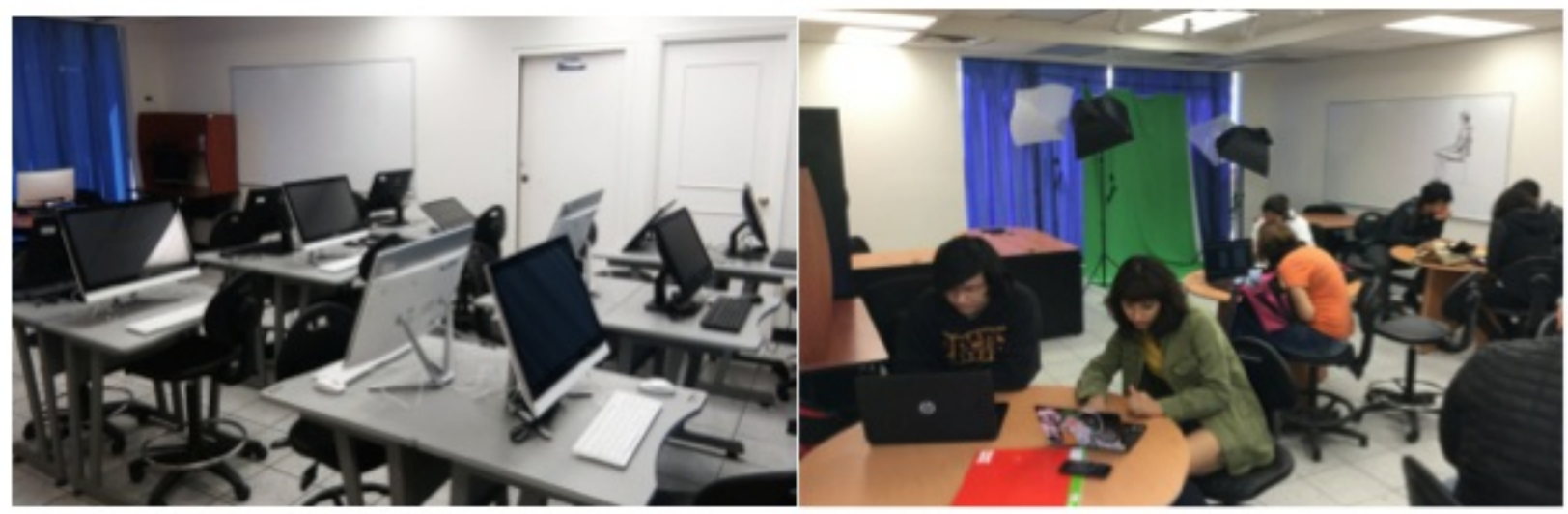

Figura No. 3 y 4 . Área de cómputo y Área "Maker" del DigitLAB.

A continuación se presenta la interface de la plataforma del DigitLAB a través de la cual se realizan proyectos y se propicia la comunicación interactiva: 


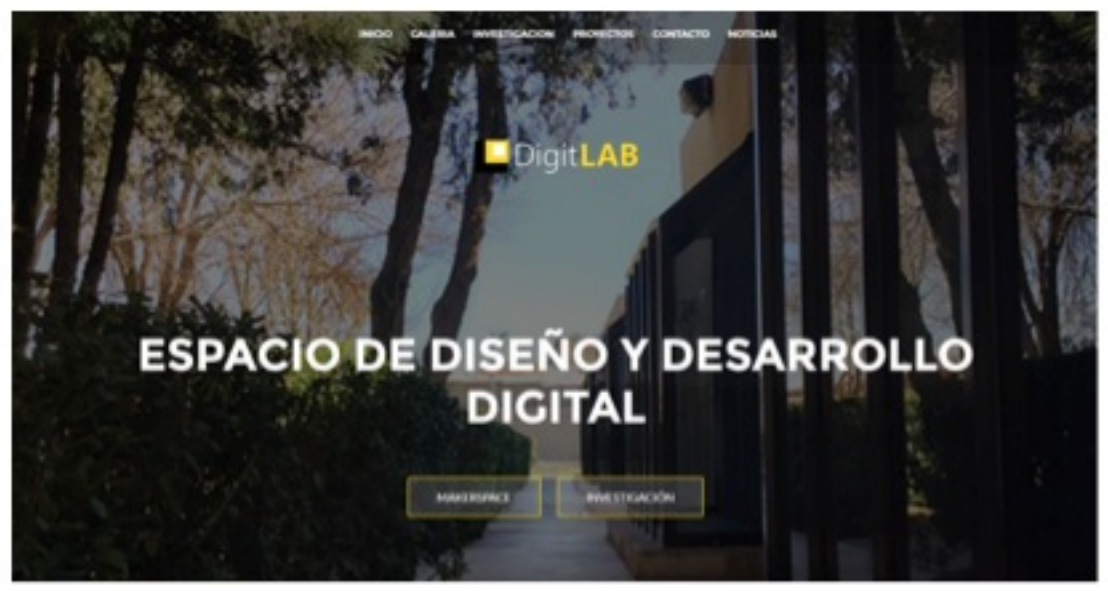

Figura No. 5. La plataforma del DigitLAB. http://digitlab.com.mx/.

\section{- Proyectos generados en el DigitLAB}

En el DigitLAB se desarrollan diversos proyectos. Este articulo mostrará tres escenarios diferentes en los cuales se estimula la comunicación y el intercambio informativo entre participantes a través de diversas herramientas digitales con la finalidad de establecer puentes de comunicación interactiva.

Proyecto 1. Diseño de Sitios Web a través de CMS (Sistema Gestor de Contenidos) en tres cursos de prácticas profesionales.

En el actual contexto educativo del diseño gráfico la enseñanza de tecnologías digitales parece interminable, es común encontrar estudiantes con inquietudes tecnológicas que van más allá de lo que aprenden en los cursos regulares que implican tecnologías para el diseño digital y web y al mismo tiempo estudiantes que no logran aplicar los conocimientos de forma eficiente en un proyecto completo de diseño web.

Este no es un problema nuevo ni exclusivo de una institución educativa, se asegura que el desarrollo de las tecnologías es la raíz de las problemáticas en el ámbito educativo y profesional del diseño gráfico. Una encuesta realizada por Echevarría (2006) entre profesionistas, profesores, estudiantes y empresarios del ámbito del diseño, reveló la urgencia de acercar a los estudiantes de diseño gráfico a los nuevos campos laborales que proponen las tecnologías emergentes, crear oportunidades para la articulación de la teoría y la práctica, apoyarlos para la ubicación en el mercado laboral, prepararlos para la práctica inter y multidisciplinar y fortalecerlos para la gestión profesional y la comunicación.

Participantes:Con la visión puesta en lo anterior, este proyecto se centró en vincular escuela, estudiantes y campo laboral. 45 estudiantes de Diseño Gráfico realizaronsitios web dinámicos que funcionaron como portafolio y bitácora para que sus facilitadores y guías pudieran hacer el seguimiento,durante un semestre,de lo que estaban realizando en el campo laboral. Los sitios webapoyaron a los estudiantes en la comunicación de sus avances y ampliaronsu conocimiento del diseño en el área digital y el diseño web, al mismo tiempo la actividad de diseño web fortaleció sus habilidades telemáticasde gestión y comunicación.

Procedimiento:El diseño de este ambiente de aprendizaje se apegó al Modelo EAC de Jonassen (2000).

1. Contexto y Representación:En este caso el DigitLAB y la plataforma virtual del laboratorio fueron una alternativa de aprendizaje para los participantes;en el laboratorio se propició un ambiente de aprendizaje abierto y colaborativo, la plataforma virtual les proporcionó un foro de soporte, videos de ejemplos, recursos Open Source para la creación de los sitios web.

2. Manipulación del espacio: Se les facilitó equipo de cómputo, software, todas las herramientas necesarias para digitalizar y documentar su trabajo de forma libre.

3. Coaching:Los estudiantes fueron apoyados tanto en el laboratorio como de forma virtual por tres 
estudiantes con más experiencia en el desarrollo web con CMS, estos a su vez realizaban proyectos de titulación con estas plataformas.

4. Scaffolding: Los facilitadores de los tres cursos dieron seguimiento a los estudiantes a través de los sitios web dinámicos desarrollados con CMS que los estudiantes realizaron.

5. Herramientas cognitivas: Se vinculó a estudiantes a redes de desarrollo web para investigar acerca de los recursos para CMS y plataformas de descarga de recursos Open Source.

6. Herramientas de comunicación: Se creó un grupo de soporte en la red socialfacebook para estimular la interacción y un foro de soporte en la plataforma virtual, asimismo para el tráfico de documentos y recursos Open Source se utilizaron carpetas compartidas en la nube.

Ejemplo de proyectos generados por los estudiantes:
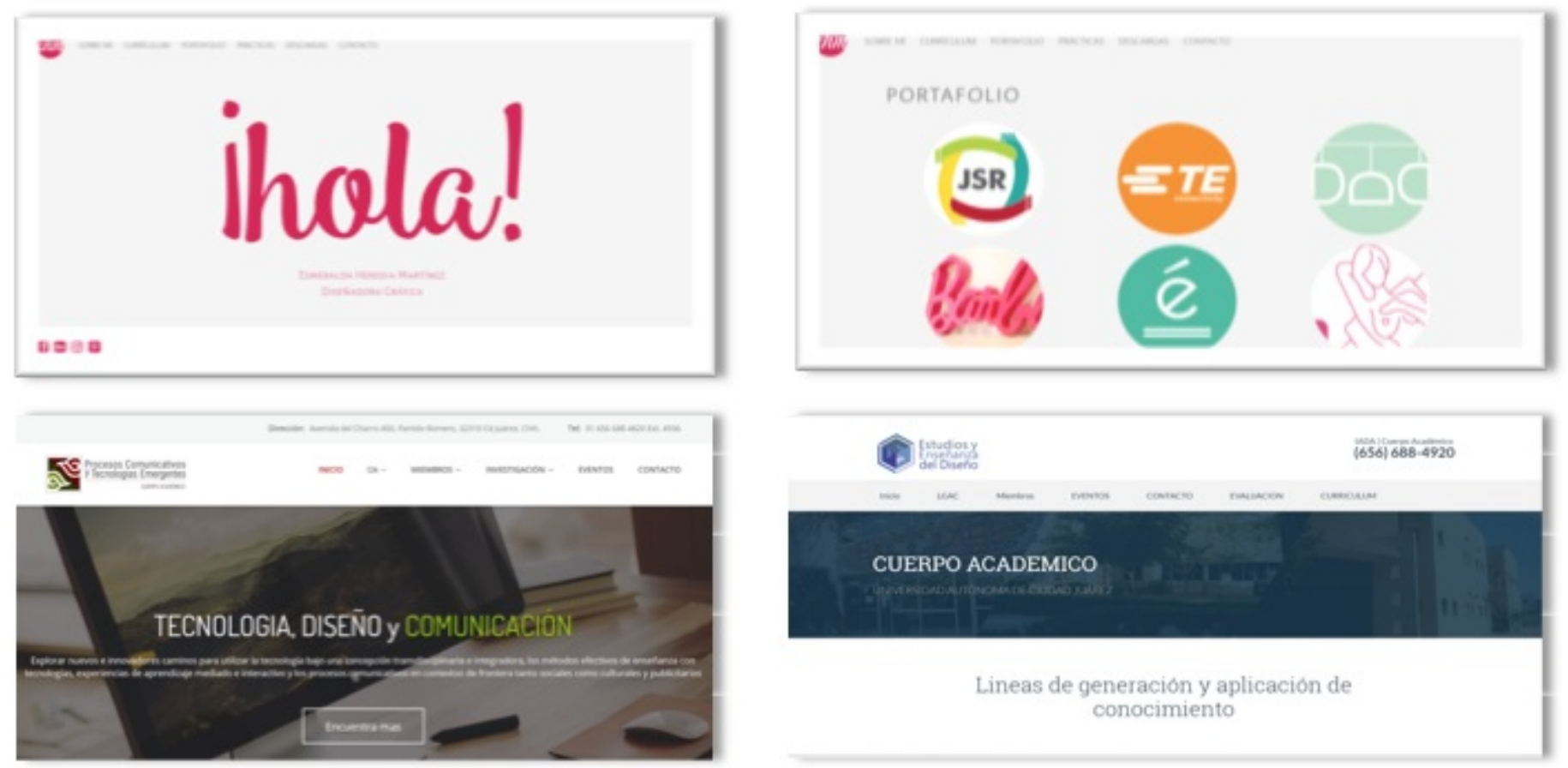

Figura No 6. Sitios web diseñados por los estudiantes.

Proyecto 2. Diseño Corporativo Interdisciplinar (Diseño gráfico y Diseño de interiores).

Un problema que aqueja a las disciplinas del diseño se refiere al tema de la interdisciplinariedad. De acuerdo a Fragoso (2008) las disciplinas del diseño se proclaman independientes, aun cuando en el campo del diseño tanto las teorías como los métodos se conceptúan desde la óptica de una actividad multidisciplinaria. Este autor asegura que por alguna razón los fenómenos del diseño, desde una perspectiva de la comunicación, se abordan con el enfoque de una sola disciplina, es decir, de manera monolítica, lineal y unilateral. Martín Juez (2002) menciona que aún nos hace falta entender la realidad como no lineal, como un conjunto de problemas que interactúan entre sí en un contexto cambiante.

El objetivo general del proyecto vinculó los cursos de Identidad y Sistemas Visuales de la licenciatura en Diseño Gráfico y Diseño Comercial y Educativo de la licenciatura en Diseño de Interiores, ambas de la Universidad Autónoma de Ciudad Juárez; el cual consistió en desarrollar una propuesta de diseño comercial o educativo de forma interdisciplinar e interconectados de forma virtual. Fue relevante involucrar ambas disciplinas en esta actividad tomando en cuenta que la realidad en la práctica profesional va por esta vertiente. En la actualidad un diseñador no solo interactúa con otras disciplinas del diseño, también participa en áreas que trascienden el ámbito del diseño, como la ingeniería, administración, ergonomía, etc.

Participantes:Para el logro de los objetivos se involucró a 60 estudiantes de cuatro cursos, dos de Diseño Gráfico y dos de Diseño de Interiores que se imparten en diferentes horarios. Los estudiantes de ambas 
disciplinas realizaron una actividad académica de ocho semanas como proyecto de fin de cursos.

Procedimiento:El diseño de este ambiente de aprendizaje se apegó al Modelo EAC de Jonassen (2000).

1. Contexto y Representación: En este caso el DigitLAB y una plataforma virtual tipo Blog realizada con tecnología Google fueron una alternativa de aprendizaje para los participantes. En el laboratorio se propició un ambiente de aprendizaje abierto y colaborativo, la plataforma virtual les proporcionó los conceptos del curso, presentación de los temas, vínculos de interés e interconexión entre equipos de trabajo.

2. Manipulación del espacio:En ambos casos, diseño de interiores y diseño gráfico, se les proporcionósala de cómputo, software, todas las herramientas necesarias para realizar el diseño. EI DigitLAB propició encuentros de trabajo interdisciplinar para el desarrollo de diseño en conjunto.

3. Coaching: Los estudiantes fueron apoyados tanto en el laboratorio como de forma virtual por sus facilitadoresen los aspectos de diseño y conceptualización.

4. Scaffolding: Los facilitadores de los cuatro cursos dieron seguimiento a los estudiantes a través del Blog del cursopara el logro de los objetivos.

5. Herramientas cognitivas:Los estudiantes utilizaron las herramientas de productividad de Google, Google Drive, Gmail, Google Fotos, Documentos, entre otras.

6. Herramientas de comunicación: Los estudiantes utilizaron herramientas de comunicación de Googlecomo Blogger, Hangouts, Google+ y Google móvil.

Ejemplo de los proyectos generados por los estudiantes:

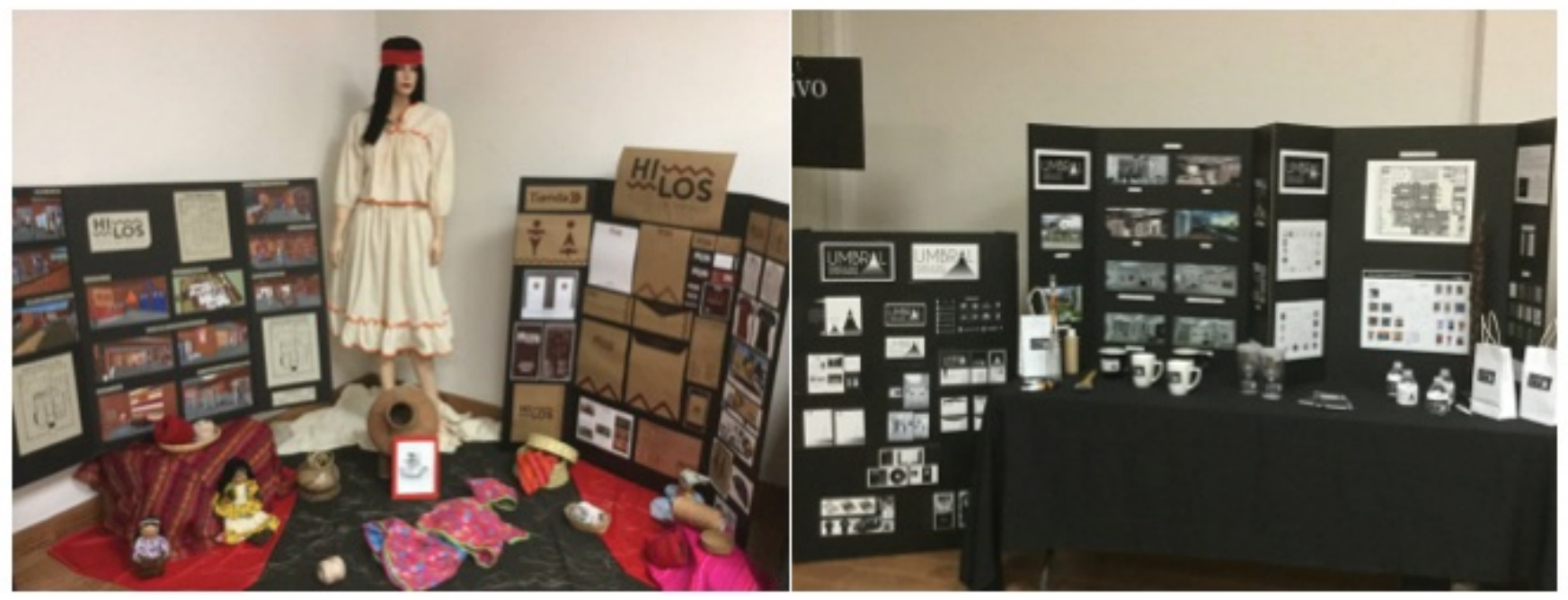

Figura No. 7 y 8 . Exposición de los proyectos de Diseño comercial y educativo desarrollados por los estudiantes de Gráfico e Interiores en forma conjunta.

Proyecto 3. Diseño de Sitios Web a través de CMS (Sistema Gestor de Contenidos) como proyectos de investigación para la titulación.

Estudiantes sin conocimientos de programación realizan sus proyectos de titulación desarrollando sitios web con enfoque social. A través de estos proyectos los estudiantes se vincularon con redes de diseño y desarrollo web para gestionar herramientas y recursos Open Source que sirvan para configurar la funcionalidadde sus sitios. Los proyectos fueron documentados en Tesinas que se presentaron para titularse como diseñadores gráficos.

Participantes:En este proyecto participaron ocho estudiantes próximos a egresar que realizaron sus proyectos de titulación en el DigitLAB, generaron vínculos con redes de conocimiento para realizar sitios web dinámicos a través de CMS'ssin conocimientos de programación. Los sitios web se realizaron atendiendo problemáticas reales con enfoque social. 
Procedimiento:

1. Contexto y Representación: En este caso el DigitLAB fue la alternativa de aprendizaje, los estudiantes participaron como guías dentro del laboratorio al mismo tiempo que realizaron sus proyectos de investigación. Asimismo colaboraron con la plataforma digital para dar soporte a sus pares. Investigaron acerca de tecnologías emergentes y recursos Open Source para la creación de los sitios web y compartieron este conocimiento con sus compañeros de prácticas profesionales para la elaboración de los portafolios y bitácoras.

2. Manipulación del espacio: Se les facilitó equipo de cómputo, software, todas las herramientas necesarias para realizar sus proyectos de investigación.

3. Coaching:Los estudiantes fueron apoyados por sus codirectores de proyecto de titulación y sus pares con más experiencia.

4. Scaffolding:A los estudiantes se les dio seguimiento durante el proyecto por el director del DigitLAB y sus directores de proyectos de titulación.

5. Herramientas cognitivas: Se vinculó a estudiantes a redes de desarrollo web para investigar acerca de los recursos para CMS y plataformas de descarga de recursos Open Source.

6. Herramientas de comunicación: Se creó un grupo de soporte en la red socialfacebook para estimular la interacción y un foro de soporte en la plataforma virtual, así mismo para el tráfico de documentos y recursos Open Source se utilizaron carpetas compartidas en la nube con las personas involucradas en los proyectos de titulación

Proyectos generados por los estudiantes: 

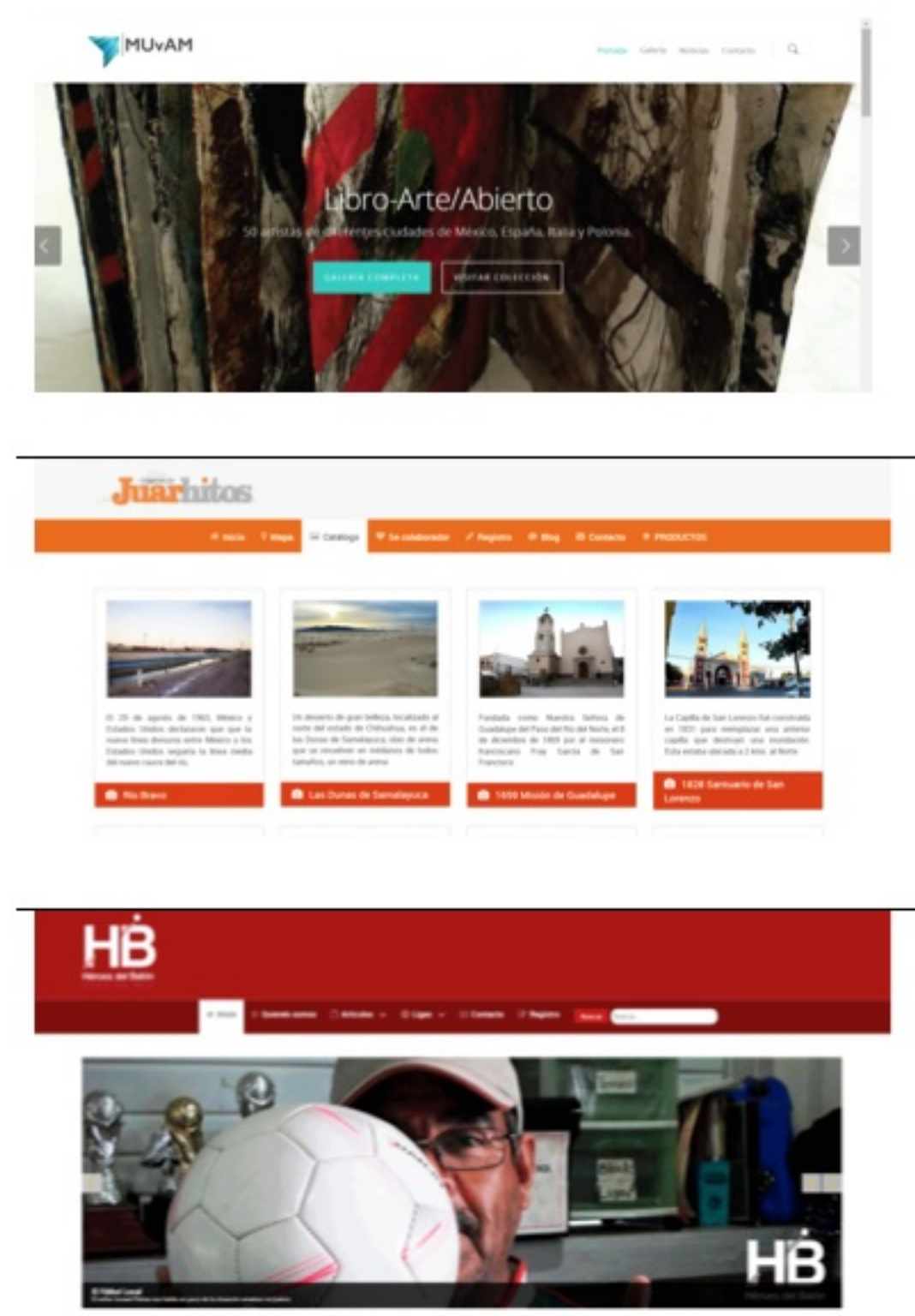

Figura No 9. Sitio webMUvAM

Libro-Arte/Abierto

Sitio para promover el arte y la cultura de Artistas de diferentes países como México, España, Italia y Polonia que exponen en esta Galería virtual.

Disponible en:

http://www.muvam.com/

Figura No 10. Sitio webJuarhitos

Galería Fotográfica urbana de Ciudad Juarez.

Sitio para promover la Fotografía Urbana.El sitio es una invitación para jóvenes fotógrafos que quieran participar para generar una imagen gráfica de Ciudad Juárez.

Disponible en:

http://www.juarhitos.com/

Figura No 11. Sitio web Héroes del Balón

Ligas de futbol amateur

Sitio para promover el futbol amateur en Ciudad Juarez.Se congregan las ligas infantiles amateur de la ciudad y se invita a jóvenes deportistas y aficionados a generar equipos, registrarlos y entrar en la competición.

Disponible en:

http://www.juarhitos.com/

\section{METODOLOGIA}

En el presente apartado se exhiben resultados cuantitativos del seguimiento semanal que se les dio a los estudiantes durante ocho semanas y que reflejan las actividades y desarrollos alcanzados en el Makerspace como ámbito educativo y en el DigitLAB como laboratorio de experiencias de comunicación y tecnologías emergentes.

\subsection{Instrumento para el análisis}

Se utilizó unaRúbrica de Evaluación del Proceso de Comunicación e interacción (Elaboración propia-Anexo) que se aplicó una vez por semana durante ocho semanas en formato de inventario. En los tres ejercicios académicos se evaluaron niveles de interacción, comunicación y adaptación al aprendizaje interdisciplinar mediado por tecnología.

\subsection{Resultados}

En este apartado se exteriorizan los resultados que arroja la aplicación del instrumento de recolección de información del presente estudio y que resulta clarificador de las actividades desarrolladas. 


\section{Interacción equipo-equipo}
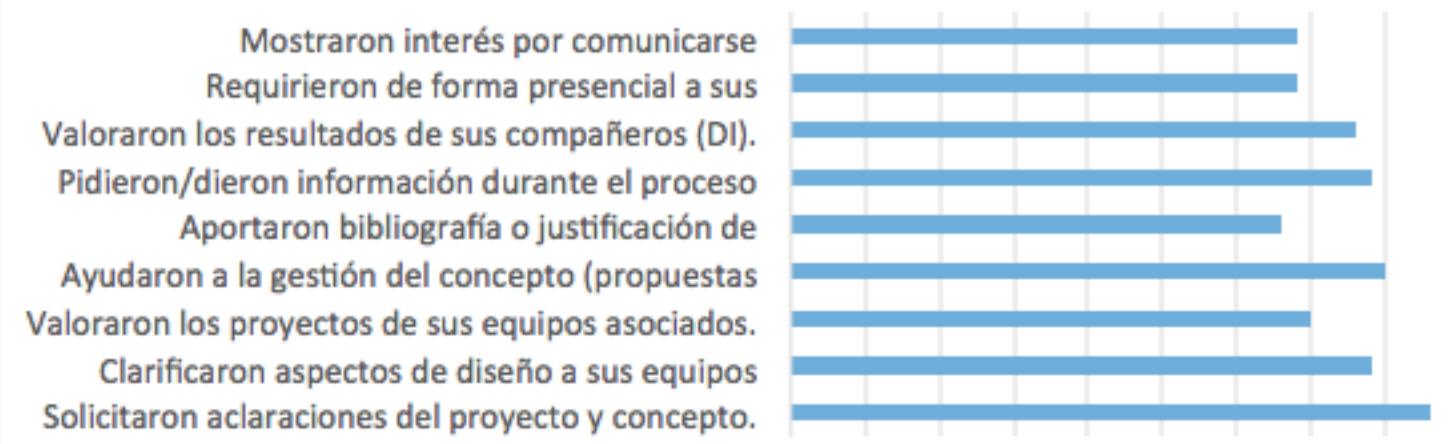

$\begin{array}{lllllllllll}0 & 0,5 & 1 & 1,5 & 2 & 2,5 & 3 & 3,5 & 4 & 4,5 & 5\end{array}$

Figura No 12. Es importante mostrar que los estudiantes de ambas disciplinas obtuvieron puntuaciones arriba de 3.5 (la puntuación mayor es 5 ) en aspectos de interacción con sus pares de otra disciplina en cuanto a compartir conceptos, diseñar y clarificar conceptos; y menor puntuación en aspectos que les implicó reunirse de forma presencial para documentar el reporte de diseño.

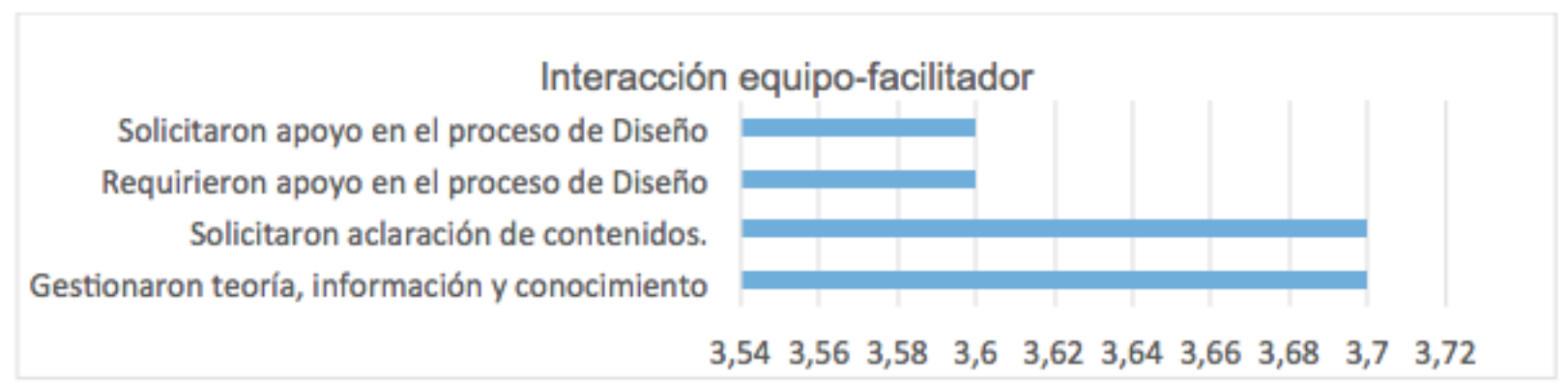

Figura No 13. Los estudiantes que trabajaron en equipos solicitaron el apoyo del facilitador no para resolver cuestiones del proceso sino para pedir aclaración de conceptos y gestionar teoría e información.

\section{Interacción facilitador-estudiante}

Propuso nuevos problemas y actividades al

Exploró las percepciones y las concepciones

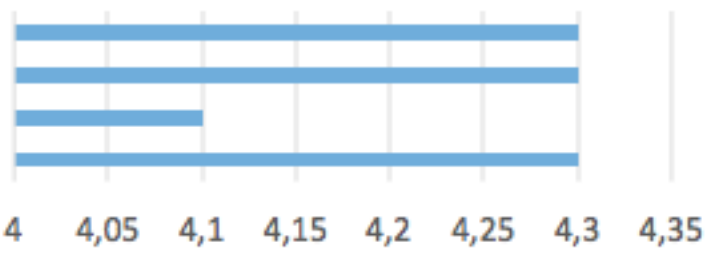

Figura No 14. En cuanto a los estudiantes que trabajaron proyectos individuales sucedió de forma contraria, requirieron más apoyo al facilitador y guías durante el proceso de diseño. 


\section{Interacción estudiante-contenidos}

Revisaron por segunda vez el contenido Indagaron más allá de lo revisado en el curso Utilizan la teoría para el desarrollo del manual Justifican diseño con la teoría Defendieron propuestas con base en la teoría

Clarifican - explican y Definen la teoría del Solicitan aclaraciones de teoría

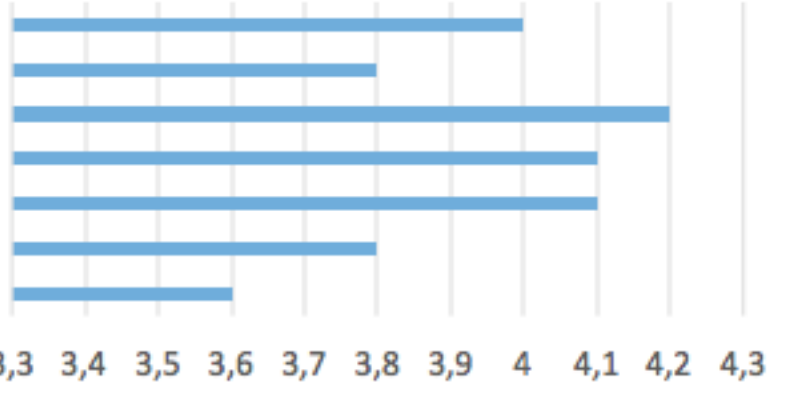

Figura No 15. En cuanto cómo los estudiantes interactuaron con los contenidos y la teoría facilitada a través de la plataforma de soporte de forma asincrónica y sincrónica, en relación a otros medios para gestionar información, fue relevante que se apegaran a lo proporcionado en las plataformas y no indagaran y clarificaran conceptos a través de otros medios como internet.

\section{Interacción estudiante-medios}

Utilizaron más de una herramienta del sistema Sugieren formas de gestionar el sistema. Informan respecto del sistema. Preguntan algún aspecto del funcionamiento del

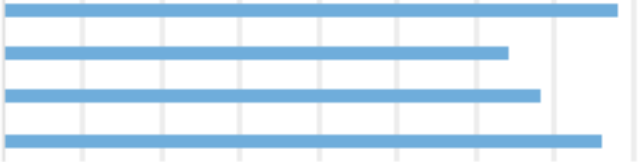

$\begin{array}{llllllllll}0 & 0,5 & 1 & 1,5 & 2 & 2,5 & 3 & 3,5 & 4 & 4,5\end{array}$

Figura No 16. En cuanto cómo los estudiantes utilizaron los medios de comunicación proporcionados a través de la plataforma y las herramientas urbanas (Google, Dropbox, Facebook, etc.), los estudiantes se mostraron interesados en utilizar la herramientas y preguntar aspectos de su funcionamiento para incluirlas en sus procesos de trabajo.

\section{Facilitador y guía a través de los medios}

Intervienen, gestionan y dan por finalizada la

Informa de nuevos recursos.

Gestionó un acuerdo con el grupo asociado

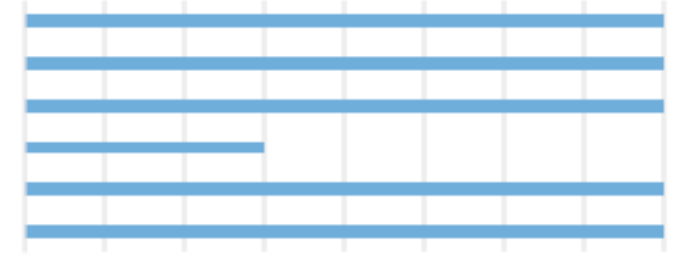

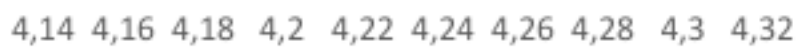

Figura No 17. En cuanto los facilitadores y guías, se vieron explicando, impartiendo pequeños cursos, explicando el sistema y comunicándose con los facilitadores de los otros cursos.

\section{CONCLUSIONES}

La alfabetización en Tecnologías de la Información y la Comunicación (TIC's) debe ser una meta educativa en las disciplinas del diseño, es evidente la necesidad de avanzar hacia nuevas formas de enseñar la tecnología propiciando espacios donde los estudiantes logren hacer la síntesis de sus conocimientos aprendiendo con y a través de tecnología. 
Asimismo,generar nuevos esquemas de interacción puede ampliar los alcances de las disciplinas del diseño, esto se puede lograr a través del proyecto académico que cada vez exige mayor contacto con la realidad profesional y la tecnología. Hoy en día es parte de esa realidad por lo tanto sus potencialidades no pueden ser ignoradas.

De acuerdo a sus propias palabras fue enriquecedor para estos estudiantes encontrar un espacio que les apoyara para trabajar con libertad, personas con inquietudes afines comprometidas y motivadas, además les resultaron relevantes las formas en que se utilizaron las herramientas y medios para la comunicación y soporte cuando así lo requirieron.

Todos se mostraron contentos de participar para alcanzar la Meta del DigitLAB en un Makerspace. En general los estudiantes transitaron de forma natural a través del proceso de diseño, ya que regularmente se desenvuelven en una nueva forma de sociedad de información y conocimiento, lo que trae consigo diversificar sus formas de aprender diseño, lo cual no significó un proceso de difícil adaptación.

\section{AGRADECIMIENTO:}

Agradecemos a la Secretaría de Educación Pública y el Programa para el Desarrollo Profesional Docente (PRODEP) por el apoyo económico otorgado para el financiamiento de este proyecto y a la Universidad Autónoma de Ciudad Juárez, el Departamento de Diseño y el Programa de Diseño Gráfico por el apoyo y facilidades prestadas para la implementación del DigitLAB.

\section{REFERENCIAS BIBLIOGRÁFICAS}

BLIKSTEIN, P. \&KRANNICH, D.(2013).The maker's movement and FabLabs in education: experiences, technologies, and research.En Proceedings of the 12th international conference on interaction design and children (IDC '13),pp. 613-616.

ECHEVERRÍA, R. (2006, octubre). Equipos de Alto Desempeño: Un modelo de intervención. Ponencia presentada en el Seminario “¿Cómo llevar a su Equipo al Alto Desempeño?”. Escuela de Psicología, Universidad Adolfo Ibáñez, Santiago, Chile.

FRAGOSO, O. (2008). "EI Diseño como actividad multidisciplinaria”. EnRevista del Centro de Investigación . Universidad La Salle, vol. 8, Núm. 29,pp. 55-68.

ITO, M., GUTIÉRREZ, K., LIVINGSTONE, S., PENUEL, B., RHODES, J., SALEN, K. \& WATKINS, S. C. (2013). Connected learning: An agenda for research and design.Digital Media and Learning Research Hub.

JONASSEN, D. (2000). El Diseño de entornos constructivistas de aprendizaje. En: Reigeluth, Ch. (eds.) Diseño de la instrucción Teorías y modelos. Un paradigma de la teoría de la instrucción.Parte I. Madrid: Aula XXI Santillana,pp. 225-249.

KNOBEL, M. \& LANKSHEAR, C. (2010).DIY Media: A Contextual Background and Some Contemporary Themes.Michele Knobel and Colin Lankshear (Eds).In DIY Media: Creating, Sharing and Learning With New Technologies.New York: Peter Lang, pp.1-26.

LEWIS, J. (2015). “Libraries Are for Making: Robots". Young Adult Library Services, 13(2), 25.

MARTÍN-JUEZ, F. (2002).Contribuciones para una antropología del diseño. D.F., México: Editorial Gedisa, S. A.

TESCONI, S., \& ARIAS, L. (2015, August). "The Transformative Potential of Making in Teacher Education: A Case Study on Teacher Training Through Making and Prototyping". In International ConferenceonDistributed, Ambient, and PervasiveInteractions. Springer International Publishing, pp. 119-128. 
TRESSERRAS, J. (2015).Diseño e Interdisciplinariedad. Una visión. [Consultado el 17 de junio de 2016] http://www.raco.cat/index.php/Waterfront/article/viewFile/2884-99/376673.

VOSSOUGHI, S., \& BEVAN, B. (2014).Making and tinkering: A review of the literature. National Research Council Committee on Out of School Time STEM, pp. 1-55.

\section{ANEXO:}

\section{Rubrica de Evaluación del proceso de Comunicación e Interacción}

\begin{tabular}{|c|c|c|c|c|c|c|}
\hline \multicolumn{2}{|c|}{$\begin{array}{l}\text { Otorga un valor a cada rubro en un valor del } 5 \text { al } 1 \\
5 \text { VA HACLA LOS POSITIVOS, } 1 \text { VA HACLA LOS NEGATIVOS }\end{array}$} & \multicolumn{3}{|c|}{$\begin{array}{l}\text { Totalmente de } \\
\text { acuerdo } \\
\end{array}$} & \multicolumn{2}{|c|}{$\begin{array}{r}\text { Totalmente en } \\
\text { desacuerdo }\end{array}$} \\
\hline & & 5 & 4 & 3 & 2 & 1 \\
\hline Categoria & Subcategoria & & & & & \\
\hline \multirow{9}{*}{ Equipo - Equipo } & Solicitaron aclaraciones del proyecto y concepto. & & & & & \\
\hline & Clarificaron aspectos de diseño a sus equipos asociados. & & & & & \\
\hline & Valoraron los proyectos de sus equipos asociados. & & & & & \\
\hline & $\begin{array}{l}\text { Ayudaron a la gestión del concepto (propuestas de } \\
\text { nombres, slogams, ideas, etc.) }\end{array}$ & & & & & \\
\hline & Aportaron bibliografia o justificación de propuestas & & & & & \\
\hline & Pidieron/dieron información durante el proceso & & & & & \\
\hline & Valoraron los resultados de sus companeros (DI). & & & & & \\
\hline & Requirieron de forma presencial a sus compañeros (DI). & & & & & \\
\hline & Mostraron interés por comunicarse & & & & & \\
\hline \multirow{4}{*}{ Equipo - Facilitador } & Gestionaron teoria, información y conocimiento de Diseño & & & & & \\
\hline & Solicitaron aclaración de contenidos. & & & & & \\
\hline & Requirieron apoyo en el proceso de Diseño & & & & & \\
\hline & Solicitaron apoyo en el proceso de Diseño & & & & & \\
\hline \multirow{7}{*}{ Estudiante Contenido } & Solicitan aclaraciones de teoría & & & & & \\
\hline & Clarifican - explican y Definen la teoria del diseño & & & & & \\
\hline & Defendieron propuestas con base en la teoría & & & & & \\
\hline & Justifican diseño con la teoria & & & & & \\
\hline & Utilizan la teoria para el desarrollo del manual & & & & & \\
\hline & Indagaron más allá de lo revisado en el curso & & & & & \\
\hline & Revisaron por segunda vez el contenido expuesto $\left({ }^{*}\right)$ & & & & & \\
\hline \multirow{4}{*}{ Estudiante Medio } & Preguntan algún aspecto del funcionamiento del sistema. & & & & & \\
\hline & Informan respecto del sistema. & & & & & \\
\hline & \begin{tabular}{|l|l|} 
Sugieren formas de gestionar el gistema. \\
\end{tabular} & & & & & \\
\hline & Utilizaron más de una herramienta del sistema & & & & & \\
\hline \multirow{4}{*}{ Facilitador estudiante } & $\begin{array}{l}\text { Exploró las percepciones y las concepciones de los } \\
\text { estudiantes para mejorar los diseños }\end{array}$ & & & & & \\
\hline & Clarificó facilitando la comprensión de los estudiantes. & & & & & \\
\hline & \begin{tabular}{|l|l|l|} 
Propuso nuevos problemas y actividades al retroalimentar \\
\end{tabular} & & & & & \\
\hline & Orientó sobre la bibliografia, gestionó y la facilitó. & & & & & \\
\hline Facilitador Facilitador & Gestionó un acuerdo con el grupo asociado & & & & & \\
\hline \multirow{2}{*}{ Facilitador medio } & Explica sobre cómo se utiliza el gistema. & & & & & \\
\hline & Informa de nuevos recursos. & & & & & \\
\hline \multirow{3}{*}{ Guias Contenido } & Organizó el contenido del curso. & & & & & \\
\hline & Intervienen, gestionan y dan por finalizada la actividad & & & & & \\
\hline & Imparten actividades. & & & & & \\
\hline
\end{tabular}

-Recursos: Google, Wedteress, Dropbox, Facebook, Otros. 


\section{BREVE SEMBLANZA DE LAS AUTORAS}

Silvia Husted Ramos es doctora en Educación de las Ciencias, Ingenierías y Tecnologías por la Universidad de las Américas Puebla. Maestra en Diseño Holístico por la Universidad Autónoma de Ciudad Juárez. Profesorainvestigadora de Tiempo Completo de la Universidad Autónoma de Ciudad Juárez en el campo del diseño gráfico y la tecnología. Intereses de investigación: Ambientes de aprendizaje efectivos para la enseñanza del diseño y creatividad aplicada.

Gloria Olivia Rodríguez Garay es doctora en Periodismo y Sociedad por la Universidad de Sevilla. Maestra en Industria Audiovisual en el Espacio Iberoamericano por la Universidad Internacional de Andalucía. Profesorainvestigadora de Tiempo Completo de la Universidad Autónoma de Ciudad Juárez en diversos campos de la comunicación. Intereses de investigación: Producción y narrativa audiovisual, lenguaje multimedia y comunicación interactiva.

Martha Patricia Álvarez Chávez es doctora en Periodismo y Sociedad por la Universidad de Sevilla. Maestra en Industria Audiovisual en el Espacio Iberoamericano por la Universidad Internacional de Andalucía. Profesorainvestigadora de Tiempo Completo de la Universidad Autónoma de Ciudad Juárez en diversos campos de la comunicación. Intereses de investigación: Producción y narrativa audiovisual, producción multimedia y comunicación interactiva, publicidad.

Ámbitos. Revista Internacional de Comunicación, n.35, edición de invierno, 2016-2017. Recibido:

Aprobado: 